\title{
Gastrointestinal Basidiobolomycosis: An Emerging Fungal Infection of the Gastrointestinal Tract, the Royal Hospital (Sultanate of Oman) Experience
}

\author{
Mohammed Al-Masqari ${ }^{1}$, Amal Al-Maani ${ }^{2}$, Fatma Ramadhan ${ }^{3}$
}

\begin{abstract}
Aims and objectives: In this study, we aimed to examine the epidemiology, clinical characteristics, histopathology findings, management, and ancillary techniques which are important for diagnosis, management, and outcome of an uncommon manifestation caused by the Basidiobolus ranarum.

Materials and methods: This is a case series of five interesting cases of gastrointestinal basidiobolomycosis (GIB). This study was conducted in the histopathology department at the Royal hospital which is a tertiary care institution. Cases diagnosed with all types of fungal gastrointestinal disease between 2008 and 2015 were reviewed. Cases with morphological features of basidiobolomycosis were retrieved and the diagnosis was confirmed by a senior pathologist.

Results: Five cases were identified. Out of five patients, four were misdiagnosed with other types of fungal infections which resulted in high morbidity and mortality. This case series revealed that the majority of patients identified are pediatrics (60\%). Further, (60\%) were from the same region (Ad Dakhiliyah). All patients presented with unspecific gastrointestinal symptoms that clinically mimic serious diseases. Additionally, all patients shared similar radiological findings and laboratory investigations.

Conclusion: Diagnosis of GIB requires a high index of suspicion, increased awareness of this rare disease aid in early diagnosis and promote an early start of treatment. Since there is a resemblance in the clinical features of inflammatory and neoplastic bowel disease, GIB should be considered in the differential diagnosis.

Keywords: Fungal infectious, Gastroenterology, Gastrointestinal basidiobolomycosis, Microbiology, Pathology.

Pediatric Infectious Disease (2021): 10.5005/jp-journals-10081-1290
\end{abstract}

\section{INTRODUCTION}

Basidiobolomycosis is a rare fungal disease caused by Basidiobolus ranarum, a fungus belonging to the family of Entomophthoraceae of the class Zygomycetes. It was first described by Eidam was the first to describe Basidiobolomycosis as an isolate from frogs in 1886 and was later cultured from intestinal contents and the excreta of frogs. ${ }^{1}$ Infection by this fungus rarely affects the gastrointestinal tract but mainly causes cutaneous disease involving the limbs, trunk, and buttocks. ${ }^{1}$ It is presumed that infection is acquired after minor trauma to the skin or insect bites. The causative agent is common in soil, decaying vegetable matter, and the dung of amphibians, reptiles, fish, and insectivorous.

Lack of awareness of gastrointestinal basidiobolomycosis (GIB) has resulted in its delayed diagnosis and extensive morbidity and mortality in patients with GIB. Awareness of this disease will result in early diagnosis and hence will prevent unfavorable outcomes such as a reduction in unnecessary surgeries and likely reduction in mortality. ${ }^{2}$ This research will be the first and the largest case serious to be done in Oman and will add to the insufficient literature published globally.

In this study, we aimed to investigate the epidemiology, clinical characteristics, histopathology findings, management, and ancillary techniques which are important for diagnosis, management, and outcome of an uncommon manifestation caused by the B. ranarum.

\section{Materials and Methods}

All the cases of GI fungal infection from 2008 to 2015 were identified through the medical record of the Royal Hospital (Al-Shifa System)

\footnotetext{
1,3 Department of Histopathology, The Royal Hospital, Muscat, Sultanate of Oman

${ }^{2}$ Department of Pediatrics, The Royal Hospital, Muscat, Sultanate of Oman

Corresponding Author: Mohammed Al-Masqari, Department of Histopathology, The Royal Hospital, Muscat, Sultanate of Oman, Phone: +968-24599617, e-mail: mohd.masqari@gmail.com

How to cite this article: Al-Masqari M, Al-Maani A, Ramadhan F. Gastrointestinal Basidiobolomycosis: An Emerging Fungal Infection of the Gastrointestinal Tract, the Royal Hospital (Sultanate of Oman) Experience. Pediatr Inf Dis 2021;3(1):46-49.

Source of support: Nil

Conflict of interest: None
}

and were reviewed by a histopathology resident and a senior consultant pathologist.

The cases of GIB are identified based on the characteristic histopathologic appearance of broad, thin-walled, pleomorphic hyphae surrounded by an eosinophilic material (known as the Splendore-Hoeppli phenomenon) (Fig. 1). Because this is a rare disease; a limited number of studies were conducted including small sample sizes. ${ }^{3-5}$ Hence, the sample size $(n=5)$ for this research is deemed acceptable. Data collected included demographic information, clinical presentation, abnormal laboratory findings, sites of involvement, radiologic studies, treatment, and outcome. Data were revised by a senior pathologist and an infectious disease consultant before the analysis. This study has been approved by the 


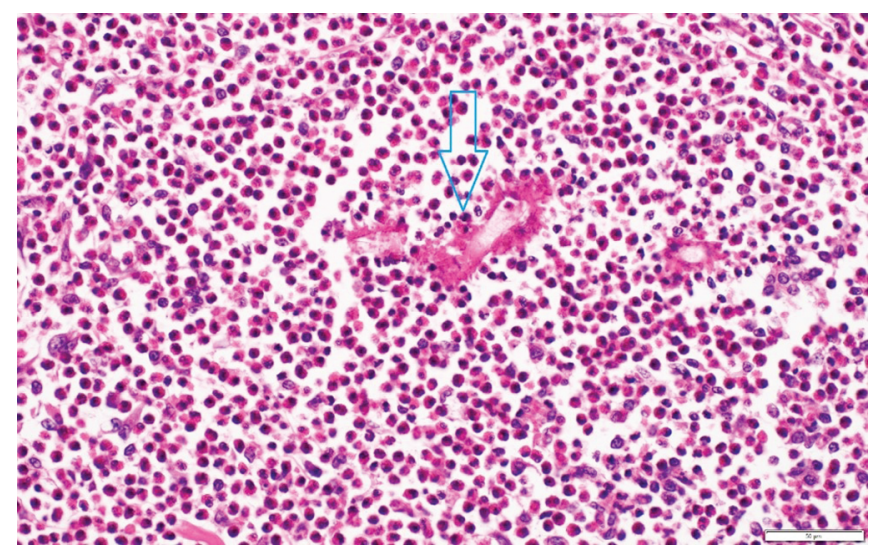

Fig. 1: Broad, thin-walled hyphae surrounded by a collar of eosinophilic material (known as the Splendore-Hoeppli phenomenon)

Clinical Research Ethics Committee of the Royal Hospital, Ministry of Health, Sultanate of Oman (Table 1).

\section{Case Descriptions}

\section{Case 1}

A 9-year-old boy presented to the regional hospital with abdominal pain, abdominal mass associated with vomiting and diarrhea. This was diagnosed as acute appendicitis after which the patient had an appendectomy which showed no significant pathological changes. Laboratory investigations showed microcystic hypochromic anemia, mild leukocytosis, thrombocytosis, and high C-reactive protein (CRP). A few weeks post-surgery, the patient developed severe abdominal pain and was referred to the Royal Hospital where CT abdomen showed a large loculated mass in the right iliac fossa. The findings were suggestive for neoplastic process. Laparotomy was done to remove the inflammatory mass which intraoperatively showed abscess. The tissue was sent for histopathological examination which showed a dense inflammatory infiltrate composed of many eosinophils and some large fungal hyphae surrounded by the Splendore-Hoeppli phenomenon. The final diagnosis was mucormycosis. The patient was treated with antibiotics but IV voriconazole was not started as it was not available at that time. The patient died due to severe fungal sepsis, septic shock, and multi-organ failure. This study revealed that this case was misdiagnosed as mucormycosis when the actual diagnosis is GIB.

\section{Case 2}

A 19-year-old girl presented to the Royal Hospital with abdominal pain, productive cough, vomiting, and fever. The first clinical impression was pneumonia as the chest $X$-ray showed a large chest mass. Laboratory investigations showed microcystic hypochromic anemia, mild leukocytosis, thrombocytosis, and high CRP. The chest mass was biopsied and the histopathological examinations showed infiltration by lymphocyte and eosinophils, thick branching fungal hyphae within pink eosinophilic materials, and the given diagnosis was mucormycosis. CT abdomen showed diffuse gastric, small intestinal, large intestinal wall thickening. The patient was treated with antibiotics and antifungal (Caspofungin, Ambisome). The patient died due to severe sepsis, disseminated fungal infection, metabolic acidosis, and hyperkalemia. This study revealed that this case was misdiagnosed as mucormycosis when the actual diagnosis is GIB.

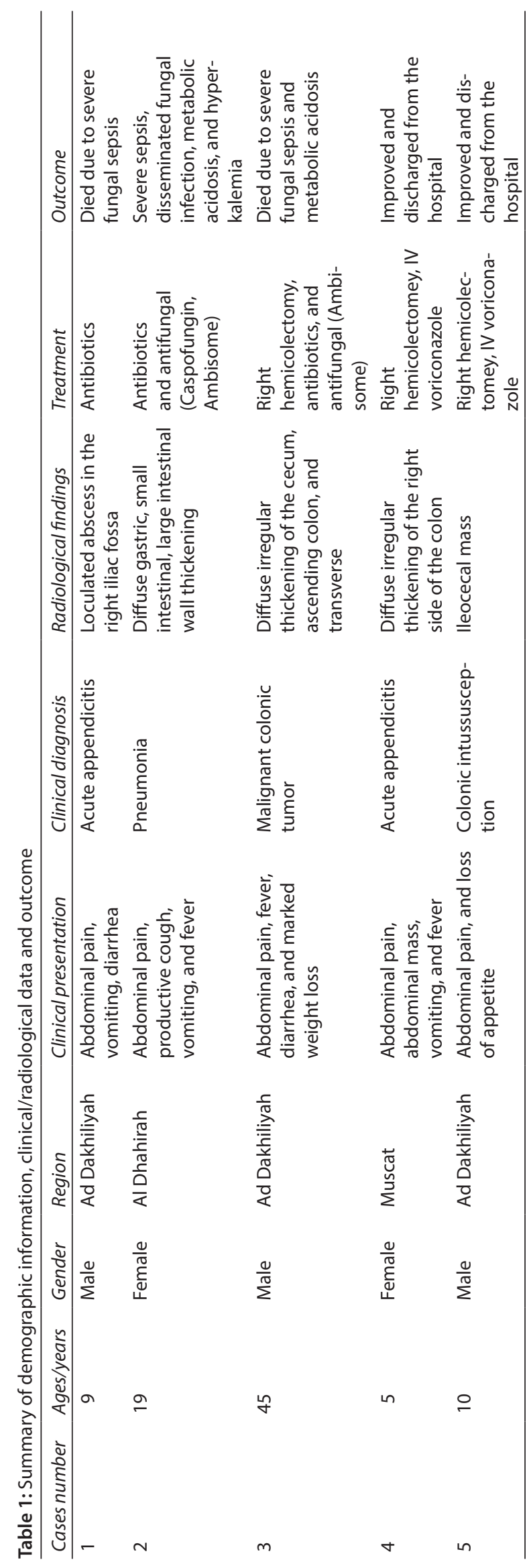




\section{Case 3}

A 45-year-old man presented to the local hospital with abdominal pain, abdominal mass, fever, diarrhea, and marked weight loss. The patient was treated conservatively. A few months later, the patient presented to the Royal Hospital with the same symptoms. Laboratory investigations showed microcystic hypochromic anemia, mild leukocytosis, thrombocytosis, and high CRP. CT abdomen showed diffuse irregular thickening of the cecum, ascending colon and transverse colon and the initial impression was a malignant colonic tumor. Right hemicolectomy was done and sent for histopathology. Intraoperatively, diffuse cecal thickening was seen. The histopathology report showed broad fungal hyphae surrounded by pink eosinophilic materials with marked eosinophils, neutrophils, and necrosis and the diagnosis was mucormycosis. The patient was treated with antibiotics and antifungal (Ambisome). The patient died due to severe fungal sepsis and metabolic acidosis. This study revealed that this case was misdiagnosed as mucormycosis when the actual diagnosis is GIB.

\section{Case 4}

A 5-year-old girl presented to the local hospital with abdominal pain, abdominal mass, vomiting, and fever. Acute appendicitis was the first clinical diagnosis and was treated with appendectomy which showed no significant pathological changes. Two weeks later, the same symptoms recurred, and the patient presented to the Royal Hospital where CT abdomen showed diffuse irregular thickening of the right side of the colon. At this point, the impression was inflammatory bowel disease vs malignancy like lymphoma or adenocarcinoma. Laboratory investigations showed microcystic hypochromic anemia, mild leukocytosis, thrombocytosis, and high CRP. Right hemicolectomy was performed to show diffuse thickening of the bowel wall and sent for histopathology which shows marked neutrophilic, eosinophilic infiltrate, and broadband fungal hyphae surrounded by eosinophilic material, and the histopathological diagnosis was mucormycosis. The patient was initially treated with antibiotics, antifungal (Ambisome) but no improvement was evident. Due to the ambiguity of the clinical and radiological symptoms, the slides were sent abroad for a second opinion and the case was diagnosed as basidiobolomycosis. Hence, IV voriconazole was added and the patient showed marked clinical improvement within a few days and the patient was discharged from the hospital with oral voriconazole. This case was the first to be diagnosed and treated successfully in the Royal Hospitals. ${ }^{6}$

\section{Case 5}

A 10-year-old boy presented to a private hospital with abdominal pain, abdominal mass and loss of appetite, and normal temperature. The initial diagnosis was colonic intussusception, and the patient was referred to the Royal Hospital for further evaluation and management. Laboratory investigations at the Royal Hospital showed microcystic hypochromic anemia with normal eosinophilic count and normal leukocytes. The CT abdomen showed an ileocecal mass (Fig. 2). Right hemicolectomy was performed and show thickening of the cecum and sent for histopathology which showed marked inflammation with marked eosinophilic infiltration, granuloma, fungal hyphae surrounded by eosinophilic material. The histopathological diagnosis was GIB. The patient was immediately treated with IV voriconazole. The patient showed marked clinical improvement and discharged on oral voriconazole. This was the second successful treatment of GIB in the Royal Hospital following the first accurate diagnosis of the case.

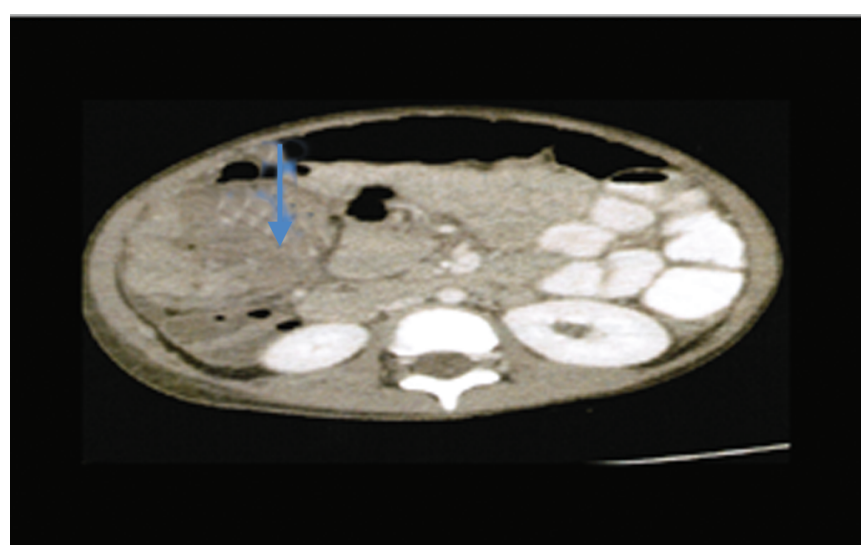

Fig. 2: Large right-sided colonic mass

\section{Discussion}

Gastrointestinal basidiobolomycosis is a rare fungal infection affecting immunocompetent hosts. As this fungus is an environmental saprophyte and present in soil and decaying vegetables and fruits, the routes of infection are most likely to be ingestion of contaminated soil, feces, or food. ${ }^{7}$ The small number of reported cases of GIB is probably related to the limited knowledge of this disease pattern. Gastrointestinal basidiobolomycosis may be rising as a result of various environmental and demographic factors. This study supports this argument as four of our patients are from the anterior regions where the weather is more warm and humid, which is a good environment for fungal growth. Furthermore, the majority of patients were children and children are more exposed to the soil as they enjoy outdoor activities; making them at higher risk of getting the infection. Gastrointestinal basidiobolomycosis should be suspected in the differential diagnosis of an immunocompetent patient presented with abdominal pain, abdominal mass, and fever with or without eosinophilia. The morbidity and mortality were very high in the previously reported cases since it may pretense as other clinical entities like inflammatory bowel disease and malignant tumor delaying the definitive diagnosis and treatment. ${ }^{3,4}$ Many factors cause difficulties in reaching the diagnosis of GIB, such as the non-specific clinical presentation, absence of predisposing or risk factors in patients, nonrepresentative colonoscopic biopsies due to involvement of nonmucosal layers of the gastrointestinal tract, and nonspecific inflammation or granulomatous reaction, with possible overlooking of the fungal hyphae. The differential diagnosis of basidiobolomycosis is mucormycosis. The later shows large, nonsepta hyphae with $90^{\circ}$ angle branching and non-parallel walls, angioinvasive causing tissue necrosis and hemorrhage. However, basidiobolomycosis usually exhibits broad, thin-walled, septate hyphae surrounded by a collar of eosinophilic material (known as the Splendore-Hoeppli phenomenon).

Awareness of this disease amongst both clinicians and pathologists helped successful treatment of the more recent cases which resulted in a marked reduction in the morbidity and mortality rates of this disease. This research is highly important as it adds to the existing literature which aids in improving service delivery and precision of care. The cases included in this study were identified through the Al-Shifa system in the Royal Hospital, therefore, the evidence is limited to the Royal Hospital. Further investigation is required to explore the risk factors that 
lead to GIB. Additionally, future research that encompasses other hospitals within Oman is suggested to examine a larger sample size.

\section{References}

1. Okafor J, Testrake D, Mushinsky $\mathrm{H}$, et al. A Basidiobolus sp. and its association with reptiles and amphibians in Southern Florida. Med Mycol 1984;22(1):47-51. DOI: 10.1080/00362178485380081.

2. Rabie M, El Hakeem I, Al-Shraim M, et al. Basidiobolomycosis of the colon masquerading as stenotic colon cancer. Case Rep Surg 2011;2011:1-3. DOI: 10.1155/2011/685460.

3. Saadah O, Farouq M, Daajani N, et al. Gastrointestinal basidiobolomycosis in a child; an unusual fungal infection mimicking fistulising Crohn's disease. J Crohn's Colitis 2012;6(3):368-372. DOI: 10.1016/j.crohns.2011.10.008.

4. Vikram H, Smilack J, Leighton J, et al. Emergence of gastrointestinal basidiobolomycosis in the United States, with a review of world wide cases. Clin Infect Dis 2012;54(12):1685-1691. DOI: 10.1093/cid/cis250.

5. Eghbalkhah A, Habibi M, Lesanpezeshki M, et al. Pediatric gastrointestinal basidiobolomycosis. Pediat Infect Dis 2016;8(1):3437. DOI: 10.1016/j.pid.2016.03.008.

6. Al-Maani AS, Paul G, Jardani A, et al. Gastrointestinal basidiobolomycosis: first case report from Oman and literature review. Sultan Qaboos Univers Med J 2014;14(2):e241-4.

7. Davis $S$, Ellis D, Goldwater P, et al. First human culture-proven Australian case of entomophthoromycosis caused by Basidiobolus ranarum. Med Mycol 1994;32(3):225-230. DOI: 10.1080/02681219480000291. 